\title{
Filamentary Structure In The Orion Molecular Cloud
}

\author{
John Bally ${ }^{1}$, Mark Dragovan ${ }^{1}$, William D. Langer ${ }^{2}$, Antony A. Stark ${ }^{1}$, and Robert W. Wilson ${ }^{1}$ \\ ${ }^{1}$ AT\&T Bell Laboratories, Crawford Hill \\ Holmdel, New Jersey \\ ${ }^{2}$ Princeton University, Princeton N.J.
}

\begin{abstract}
We present a large scale ${ }^{13} \mathrm{CO}$ map (containing 33,000 spectra) of the giant molecular cloud located in the southern part of Orion which contains the Orion Nebula, NGC1977, and the L1641 dark cloud complex. The overall structure of the cloud is filamentary, with individual features having a length up to 40 times their width. This morphology may result from the effects of star formation in the region or embedded magnetic fields in the cloud.
\end{abstract}

The Orion region contains the nearest giant molecular clouds (GMC's) to the Sun. Located at a distance of $500 \mathrm{pc}$, these clouds are situated 10 to 14 degrees below the galactic plane toward the outer galaxy, well away from potentially confusing background clouds. We picked the southern region of Orion for a detailed ${ }^{13} \mathrm{CO}$ survey of an entire GMC. The more abundant ${ }^{12} \mathrm{CO}$ species is optically thick along most lines of sight and is best suited as a probe of the kinetic temperature near the front surface of the cloud where the optical depth is unity. The rarer ${ }^{13} \mathrm{CO}$ species is optically thin in most directions as demonstrated by $\mathrm{C}^{18} \mathrm{O}$ observations; its brightness is a probe of the column density of molecular gas through the entire cloud.

The most intense feature in the map is a $13 \mathrm{pc}$ elongated S-shaped filament located in the northern part of the cloud (Figure 1). This feature contains NGC1977 at its northern end, the Orion Nebula in the middle, and has an ill-defined southern end $20^{\prime}$ north of NGC1999. This filament is about $0.5 \mathrm{pc}$ wide, has a total mass of $5 \times 10^{3} \mathrm{M}_{\odot}$, and shows a velocity gradient which peaks behind the Orion Nebula. The middle and lower portions of the map also exhibit fragmented and filamentary emission (Figure 2). Individual channel maps show about a dozen filaments. These filaments are straight, with little or no velocity gradient and are mostly aligned with the cloud. Some of these structures exhibit shapes reminiscent of the cometary globules seen in the Gum nebula.

Simple models of gravitational collapse of rotating or magnetized clouds produce flattened disc-like structures, however, the observed morphology of the Orion-A cloud requires a more sophisticated model of cloud formation or evolution. Filaments may be formed by several mechanisms including: 1) fragmentation of expanding shells associated with HII regions, stellar winds, or supernovae; 2) shadowing of the dissociating radiation from a localized source; and 3 ) magnetic fields.

If the observed velocity gradient in the major filament is interpreted as rotation about an East-West axis, then the filament can be supported against gravitational collapse along its length by centrifugal forces. For a rotation velocity of $1.75 \mathrm{~km} \mathrm{~s}^{-1} 4.5 \mathrm{pc}$ from the center (implying a rotation period of $16 \mathrm{Myr}$ ), the computed dynamic mass (defined as the mass required to gravitationally bind the cloud) is $\mathrm{M}_{\mathrm{dyn}}=\mathrm{R}(\Delta \mathrm{V})^{2} / \mathrm{G}>3 \times 10^{3} \mathrm{M}_{\odot}$, only slightly below the total mass derived from the ${ }^{13} \mathrm{CO}$ column densities. If a magnetic field threads the length of this structure, an upper bound on the strength of such a B-field can be obtained from the condition that the cylinder of gas is magnetically supported in the transverse direction. For a cylinder with major axis radius $R$ and minor axis radius $r, B<M\left[8 G / R^{2} r^{2}\right]^{1 / 2} \approx 300 \mu G$. If turbulence plays a significant role in cloud support, the equilibrium $B$ field value may be 
considerably less.

We suggest a simple picture for the evolution of the Orion-A cloud and the formation of the major filament. A rotating proto-cloud (counter rotating with respect to the galaxy) contains a B-field aligned with the galactic plane. The northern portion of this cloud collapsed first, perhaps triggered by the pressure of the Ori I OB association. The magnetic field combined with the anisotropic pressure produced by the OB-association breaks the symmetry of the pancake instability, a filament rather than a disc is produced. The growth of instabilities in the filament formed sub-condensations which are recent sites of star formation.

Figure 1: A map showing the S-shaped, major filament in the northern part of the molecular cloud which contains OMC-1 and OMC-2. The contours show the ${ }^{13} \mathrm{CO}$ antenna temperature $T_{\mathrm{A}}^{*}$, integrated from $\quad V_{L S R}=7 \mathrm{~km} \mathrm{~s}^{-1}$ to $V_{\text {LSR }}=13 \mathrm{~km} \mathrm{~s}^{-1}$ in steps of 5 $\mathrm{Kkm} \mathrm{s}^{-1}$.

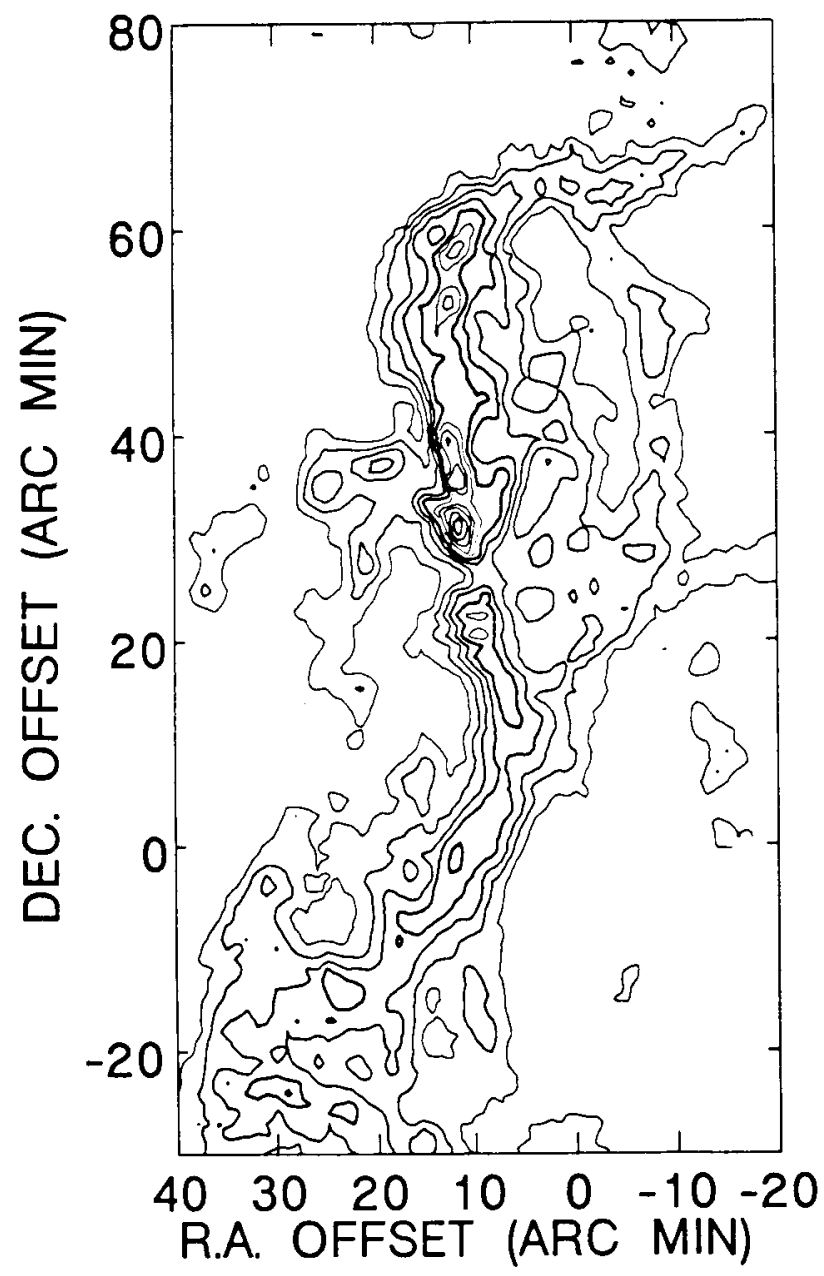

Figure 2: A map showing one of the "cometary" filaments located in the southern portion of the Orion-A molecular cloud. The contours show the ${ }^{13} \mathrm{CO}$ antenna temperature integrated from $V_{\mathrm{LSR}}=5.5 \mathrm{~km} \mathrm{~s}^{-1}$ to $\mathrm{V}_{\mathrm{LSR}}=7.5 \mathrm{~km} \mathrm{~s}^{-1}$. Contours are in $2.5 \mathrm{Kkm} \mathrm{s}^{-1}$ steps.

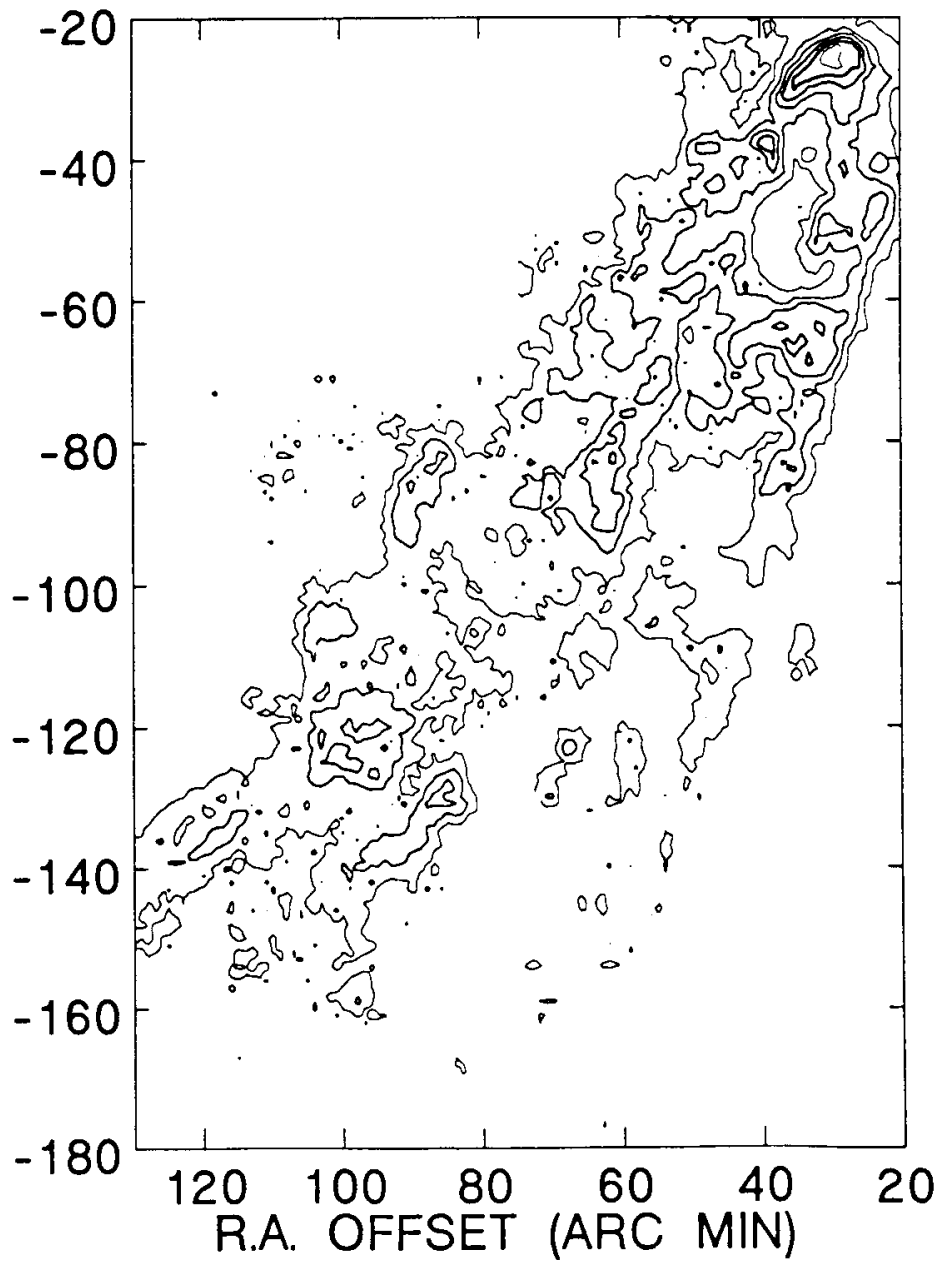

